\title{
On the Complex Symmetric and Skew-Symmetric Operators with a Simple Spectrum
}

\author{
Sergey M. ZAGORODNYUK
}

School of Mathematics and Mechanics, Karazin Kharkiv National University, 4 Svobody Square, Kharkiv 61077, Ukraine

E-mail: Sergey.M.Zagorodnyuk@univer.kharkov.ua

Received December 14, 2010, in final form February 11, 2011; Published online February 16, 2011 doi:10.3842/SIGMA.2011.016

\begin{abstract}
In this paper we obtain necessary and sufficient conditions for a linear bounded operator in a Hilbert space $H$ to have a three-diagonal complex symmetric matrix with non-zero elements on the first sub-diagonal in an orthonormal basis in $H$. It is shown that a set of all such operators is a proper subset of a set of all complex symmetric operators with a simple spectrum. Similar necessary and sufficient conditions are obtained for a linear bounded operator in $H$ to have a three-diagonal complex skew-symmetric matrix with nonzero elements on the first sub-diagonal in an orthonormal basis in $H$.
\end{abstract}

Key words: complex symmetric operator; complex skew-symmetric operator; cyclic operator; simple spectrum

2010 Mathematics Subject Classification: 44A60

\section{Introduction}

In last years an increasing interest is devoted to the subject of operators related to bilinear forms in a Hilbert space (see $[1,2,3]$ and references therein), i.e. to the following forms:

$$
[x, y]_{J}:=(x, J y)_{H}, \quad x, y \in H,
$$

where $J$ is a conjugation and $(\cdot, \cdot)_{H}$ is the inner product in a Hilbert space $H$. The conjugation $J$ is an antilinear operator in $H$ such that $J^{2} x=x, x \in H$, and

$$
(J x, J y)_{H}=(y, x)_{H}, \quad x, y \in H .
$$

Recall that a linear operator $A$ in $H$ is said to be $J$-symmetric ( $J$-skew-symmetric) if

$$
[A x, y]_{J}=[x, A y]_{J}, \quad x, y \in D(A),
$$

or, respectively,

$$
[A x, y]_{J}=-[x, A y]_{J}, \quad x, y \in D(A)
$$

If a linear bounded operator $A$ in a Hilbert space $H$ is $J$-symmetric ( $J$-skew-symmetric) for a conjugation $J$ in $H$, then $A$ is said to be complex symmetric (respectively complex skewsymmetric). The matrices of complex symmetric (skew-symmetric) operators in certain bases of $H$ are complex symmetric (respectively skew-symmetric) semi-infinite matrices. Observe that for a bounded linear operator $A$ conditions (1) and (2) are equivalent to conditions

$$
J A J=A^{*},
$$


and

$$
J A J=-A^{*},
$$

respectively.

Recall that a bounded linear operator $A$ in a Hilbert space $H$ is said to have a simple spectrum if there exists a vector $x_{0} \in H$ (cyclic vector) such that

$$
\overline{\operatorname{Lin}\left\{A^{k} x_{0}, k \in \mathbb{Z}_{+}\right\}}=H .
$$

Observe that these operators are also called cyclic operators.

It is well known that a bounded self-adjoint operator with a simple spectrum has a bounded semi-infinite real symmetric three-diagonal (Jacobi) matrix in a certain orthonormal basis (e.g. [4, Theorem 4.2.3]).

The aim of our present investigation is to describe a class $C_{+}=C_{+}(H)\left(C_{-}=C_{-}(H)\right)$ of linear bounded operators in a Hilbert space $H$, which have three-diagonal complex symmetric (respectively skew-symmetric) matrices with non-zero elements on the first sub-diagonal in some orthonormal bases of $H$. We obtain necessary and sufficient conditions for a linear bounded operator in a Hilbert space $H$ to belong to the class $C_{+}\left(C_{-}\right)$. The class $C_{+}\left(C_{-}\right)$is a subset of the class of all complex symmetric (respectively skew-symmetric) operators in $H$ with a simple spectrum. Moreover, it is shown that $C_{+}(H)$ is a proper subset.

Notations. As usual, we denote by $\mathbb{R}, \mathbb{C}, \mathbb{N}, \mathbb{Z}, \mathbb{Z}_{+}$the sets of real numbers, complex numbers, positive integers, integers and non-negative integers, respectively; $\operatorname{Im} z=\frac{1}{2 i}(z-\bar{z})$, $z \in \mathbb{C}$. Everywhere in this paper, all Hilbert spaces are assumed to be separable. By $(\cdot, \cdot)_{H}$ and $\|\cdot\|_{H}$ we denote the scalar product and the norm in a Hilbert space $H$, respectively. The indices may be omitted in obvious cases. For a set $M$ in $H$, by $\bar{M}$ we mean the closure of $M$ in the norm $\|\cdot\|_{H}$. For $\left\{x_{n}\right\}_{n \in \mathbb{Z}_{+}}, x_{n} \in H$, we write $\operatorname{Lin}\left\{x_{n}\right\}_{n \in \mathbb{Z}_{+}}$for the set of linear combinations of elements $\left\{x_{n}\right\}_{n \in \mathbb{Z}_{+}}$. The identity operator in $H$ is denoted by $E_{H}$. For an arbitrary linear operator $A$ in $H$, the operators $A^{*}, \bar{A}, A^{-1}$ mean its adjoint operator, its closure and its inverse (if they exist). By $D(A)$ and $R(A)$ we mean the domain and the range of the operator $A$. The norm of a bounded operator $A$ is denoted by $\|A\|$. By $P_{H_{1}}^{H}=P_{H_{1}}$ we mean the operator of orthogonal projection in $H$ on a subspace $H_{1}$ in $H$.

\section{The classes $C_{ \pm}(H)$}

Let $\mathcal{M}=\left(m_{k, l}\right)_{k, l=0}^{\infty}, m_{k, l} \in \mathbb{C}$, be a semi-infinite complex matrix. We shall say that $\mathcal{M}$ belongs to the class $\mathfrak{M}_{3}^{+}$, if and only if the following conditions hold:

$$
\begin{aligned}
& m_{k, l}=0, \quad k, l \in \mathbb{Z}_{+}, \quad|k-l|>1, \\
& m_{k, l}=m_{l, k}, \quad k, l \in \mathbb{Z}_{+}, \\
& m_{k, k+1} \neq 0, \quad k \in \mathbb{Z}_{+} .
\end{aligned}
$$

We shall say that $\mathcal{M}$ belongs to the class $\mathfrak{M}_{3}^{-}$, if and only if the conditions (4), (6) hold and

$$
m_{k, l}=-m_{l, k}, \quad k, l \in \mathbb{Z}_{+} .
$$

Let $A$ be a linear bounded operator in an infinite-dimensional Hilbert space $H$. We say that $A$ belongs to the class $C_{+}=C_{+}(H)\left(C_{-}=C_{-}(H)\right)$ if and only if there exists an orthonormal basis $\left\{e_{k}\right\}_{k=0}^{\infty}$ in $H$ such that the matrix

$$
\mathcal{M}=\left(\left(A e_{l}, e_{k}\right)\right)_{k, l=0}^{\infty},
$$

belongs to $\mathfrak{M}_{3}^{+}$(respectively to $\mathfrak{M}_{3}^{-}$). 
Let $y_{0}, y_{1}, \ldots, y_{n}$ be arbitrary vectors in $H, n \in \mathbb{Z}_{+}$. Set

$$
\Gamma\left(y_{0}, y_{1}, \ldots, y_{n}\right):=\operatorname{det}\left(\left(y_{k}, y_{l}\right)_{H}\right)_{k, l=0}^{n} \text {. }
$$

Thus, $\Gamma\left(y_{0}, y_{1}, \ldots, y_{n}\right)$ is the Gram determinant of vectors $y_{0}, y_{1}, \ldots, y_{n}$.

The following theorem provides a description of the class $C_{+}(H)$.

Theorem 1. Let $A$ be a linear bounded operator in an infinite-dimensional Hilbert space $H$. The operator $A$ belongs to the class $C_{+}(H)$ if and only if the following conditions hold:

(i) A is a complex symmetric operator with a simple spectrum;

(ii) there exists a cyclic vector $x_{0}$ of $A$ such that the following relations hold:

$$
\Gamma\left(x_{0}, x_{1}, \ldots, x_{n}, x_{n}^{*}\right)=0, \quad \forall n \in \mathbb{N}
$$

where

$$
x_{k}=A^{k} x_{0}, \quad x_{k}^{*}=\left(A^{*}\right)^{k} x_{0}, \quad k \in \mathbb{N} ;
$$

and $J x_{0}=x_{0}$, for a conjugation $J$ in $H$ such that $J A J=A^{*}$.

Proof. Necessity. Let $H$ be an infinite-dimensional Hilbert space and $A \in C_{+}(H)$. Let $\left\{e_{k}\right\}_{k=0}^{\infty}$ be an orthonormal basis in $H$ such that the matrix $\mathcal{M}=\left(m_{k, l}\right)_{k, l=0}^{\infty}$ belongs to $\mathfrak{M}_{3}^{+}$, where $m_{k, l}=\left(\left(A e_{l}, e_{k}\right)\right)_{k, l=0}^{\infty}$. Observe that

$$
\begin{aligned}
& A e_{0}=m_{0,0} e_{0}+m_{1,0} e_{1}, \\
& A e_{k}=m_{k-1, k} e_{k-1}+m_{k, k} e_{k}+m_{k+1, k} e_{k+1}, \quad k \in \mathbb{N} .
\end{aligned}
$$

Suppose that

$$
e_{r} \in \operatorname{Lin}\left\{A^{j} e_{0}, 0 \leq j \leq r\right\}, \quad 0 \leq r \leq n,
$$

for some $n \in \mathbb{N}$ (for $n=0$ it is trivial). By (9) we may write

$$
e_{n+1}=\frac{1}{m_{n+1, n}}\left(A e_{n}-m_{n-1, n} e_{n-1}-m_{n, n} e_{n}\right) \in \operatorname{Lin}\left\{A^{j} e_{0}, 0 \leq j \leq n+1\right\} .
$$

Here $m_{-1,0}:=0$ and $e_{-1}:=0$. By induction we conclude that

$$
e_{r} \in \operatorname{Lin}\left\{A^{j} e_{0}, 0 \leq j \leq r\right\}, \quad r \in \mathbb{Z}_{+} .
$$

Therefore $\overline{\operatorname{Lin}\left\{A^{j} e_{0}, j \in \mathbb{Z}_{+}\right\}}=H$, i.e. the operator $A$ has a simple spectrum and $e_{0}$ is a cyclic vector of $A$.

Consider the following conjugation:

$$
J \sum_{k=0}^{\infty} x_{k} e_{k}=\sum_{k=0}^{\infty} \overline{x_{k}} e_{k}, \quad x=\sum_{k=0}^{\infty} x_{k} e_{k} \in H .
$$

Observe that

$$
\left[A e_{k}, e_{l}\right]_{J}=\left(A e_{k}, e_{l}\right)=m_{l, k}=m_{k, l}=\left(A e_{l}, e_{k}\right)=\left[A e_{l}, e_{k}\right]_{J}, \quad k, l \in \mathbb{Z}_{+} .
$$

By linearity of the $J$-form $[\cdot, \cdot]_{J}$ in the both arguments we get

$$
[A x, y]_{J}=[A y, x]_{J}, \quad x, y \in H .
$$


Thus, the operator $A$ is $J$-symmetric and relation (3) holds. Notice that $J e_{0}=e_{0}$. It remains to check if relation (8) holds. Set

$$
H_{r}:=\operatorname{Lin}\left\{A^{j} e_{0}, 0 \leq j \leq r\right\}, \quad r \in \mathbb{Z}_{+} .
$$

By (10) we see that $e_{0}, e_{1}, \ldots, e_{r} \in H_{r}$, and therefore $\left\{e_{j}\right\}_{j=0}^{r}$ is an orthonormal basis in $H_{r}$ $\left(r \in \mathbb{Z}_{+}\right)$. Since $J e_{j}=e_{j}, j \in \mathbb{Z}_{+}$, we have

$$
J H_{r} \subseteq H_{r}, \quad r \in \mathbb{Z}_{+} .
$$

Then

$$
\left(A^{*}\right)^{r} e_{0}=(J A J)^{r} e_{0}=J A^{r} J e_{0}=J A^{r} e_{0} \in H_{r}, \quad r \in \mathbb{Z}_{+} .
$$

Therefore vectors $e_{0}, A e_{0}, \ldots, A^{r} e_{0},\left(A^{*}\right)^{r} e_{0}$, are linearly dependent and their Gram determinant is equal to zero. Thus, relation (8) holds with $x_{0}=e_{0}$.

Sufficiency. Let $A$ be a bounded operator in a Hilbert space $H$ satisfying conditions $(i),(i i)$ in the statement of the theorem. For the cyclic vector $x_{0}$ we set

$$
H_{r}:=\operatorname{Lin}\left\{A^{j} x_{0}, 0 \leq j \leq r\right\}, \quad r \in \mathbb{Z}_{+} .
$$

Observe that

$$
A^{r+1} x_{0} \notin H_{r}, \quad r \in \mathbb{Z}_{+} .
$$

In fact, suppose that for some $k \in \mathbb{N}$, we have

$$
A^{r+j} x_{0} \in H_{r}, \quad 1 \leq j \leq k .
$$

Then

$$
A^{r+k+1} x_{0}=A A^{r+k} x_{0}=A \sum_{t=0}^{r} \alpha_{r, k ; t} A^{t} x_{0}=\sum_{t=0}^{r} \alpha_{r, k ; t} A^{t+1} x_{0} \in H_{r}, \quad \alpha_{r, k ; t} \in \mathbb{C} .
$$

By induction we obtain

$$
A^{r+j} x_{0} \in H_{r}, \quad j \in \mathbb{Z}_{+} .
$$

Therefore $H=H_{r}$. We obtain a contradiction since $H$ is infinite-dimensional.

Let us apply the Gram-Schmidt orthogonalization method to the sequence $x_{0}, A x_{0}, A^{2} x_{0}, \ldots$. Namely, we set

$$
g_{0}=\frac{x_{0}}{\left\|x_{0}\right\|_{H}}, \quad g_{r+1}=\frac{A^{r+1} x_{0}-\sum_{j=0}^{r}\left(A^{r+1} x_{0}, g_{j}\right)_{H} g_{j}}{\left\|A^{r+1} x_{0}-\sum_{j=0}^{r}\left(A^{r+1} x_{0}, g_{j}\right)_{H} g_{j}\right\|_{H}}, \quad r \in \mathbb{Z}_{+} .
$$

By construction we have

$$
H_{r}=\operatorname{Lin}\left\{g_{j}, 0 \leq j \leq r\right\}, \quad r \in \mathbb{Z}_{+} .
$$

Therefore $\left\{g_{j}\right\}_{j=0}^{r}$ is an orthonormal basis in $H_{r}\left(r \in \mathbb{Z}_{+}\right)$and $\left\{g_{j}\right\}_{j \in \mathbb{Z}_{+}}$is an orthonormal basis in $H$.

From (8) and (11) we conclude that

$$
J A^{n} x_{0}=J A^{n} J x_{0}=\left(A^{*}\right)^{n} x_{0} \in H_{n}, \quad n \in \mathbb{Z}_{+} .
$$


Therefore

$$
J H_{r} \subseteq H_{r}, \quad r \in \mathbb{Z}_{+} .
$$

Let

$$
J g_{r}=\sum_{j=0}^{r} \beta_{r, j} g_{j}, \quad \beta_{r, j} \in \mathbb{C}, \quad r \in \mathbb{Z}_{+} .
$$

Using properties of the conjugation and relation (12) we get

$$
\beta_{r, j}=\left(J g_{r}, g_{j}\right)_{H}=\left(J g_{r}, J J g_{j}\right)_{H}=\overline{\left(g_{r}, J g_{j}\right)_{H}}=0,
$$

for $0 \leq j \leq r-1$. Therefore

$$
J g_{r}=\beta_{r, r} g_{r}, \quad \beta_{r, r} \in \mathbb{C}, \quad r \in \mathbb{Z}_{+} .
$$

Since $\left\|g_{r}\right\|^{2}=\left\|J g_{r}\right\|^{2}=\left|\beta_{r, r}\right|^{2}\left\|g_{r}\right\|^{2}$, we have

$$
\beta_{r, r}=e^{i \varphi_{r}}, \quad \varphi_{r} \in[0,2 \pi), \quad r \in \mathbb{Z}_{+} .
$$

Set

$$
e_{r}:=e^{i \frac{\varphi_{r}}{2}} g_{r}, \quad r \in \mathbb{Z}_{+} .
$$

Then $\left\{e_{j}\right\}_{j=0}^{r}$ is an orthonormal basis in $H_{r}\left(r \in \mathbb{Z}_{+}\right)$and $\left\{e_{j}\right\}_{j \in \mathbb{Z}_{+}}$is an orthonormal basis in $H$. Observe that

$$
J e_{r}=J e^{i \frac{\varphi_{r}}{2}} g_{r}=e^{-i \frac{\varphi_{r}}{2}} J g_{r}=e^{i \frac{\varphi_{r}}{2}} g_{r}=e_{r}, \quad r \in \mathbb{Z}_{+} .
$$

Define the matrix $\mathcal{M}=\left(m_{k, l}\right)_{k, l=0}^{\infty}$ by $(7)$. Notice that

$$
m_{k, l}=\left(A e_{l}, e_{k}\right)_{H}=\left[A e_{l}, e_{k}\right]_{J}=\left[e_{l}, A e_{k}\right]_{J}=\left[A e_{k}, e_{l}\right]_{J}=\left(A e_{k}, e_{l}\right)_{H}=m_{l, k},
$$

where $k, l \in \mathbb{Z}_{+}$, and therefore $\mathcal{M}$ is complex symmetric.

If $l \geq k+2\left(k, l \in \mathbb{Z}_{+}\right)$, then

$$
m_{k, l}=\left(A e_{l}, e_{k}\right)_{H}=\left[A e_{l}, e_{k}\right]_{J}=\left[e_{l}, A e_{k}\right]_{J}=\left(e_{l}, J A e_{k}\right)_{H}=0,
$$

since $J A e_{k} \in H_{k+1} \subseteq H_{l-1}$, and $e_{l} \in H_{l} \ominus H_{l-1}$. Therefore $\mathcal{M}$ is three-diagonal.

Since $e_{r} \in H_{r}$, using the definition of $H_{r}$ we get

$$
A e_{r} \subseteq H_{r+1}, \quad r \in \mathbb{Z}_{+} .
$$

Observe that

$$
A e_{r} \notin H_{r}, \quad r \in \mathbb{Z}_{+} .
$$

In fact, in the opposite case we get

$$
A e_{j} \in H_{r}, \quad 0 \leq j \leq r,
$$

and $A H_{r} \subseteq H_{r}$. Then $A^{k} x_{0} \in H_{r}, k \in \mathbb{Z}_{+}$, and $H=H_{r}$. This is a contradiction since $H$ is an infinite-dimensional space.

Hence, we may write

$$
A e_{r}=\sum_{j=0}^{r+1} \gamma_{r, j} e_{j}, \quad \gamma_{r, j} \in \mathbb{C}, \quad \gamma_{r, r+1} \neq 0 .
$$

Observe that

$$
m_{r+1, r}=\left(A e_{r}, e_{r+1}\right)_{H}=\gamma_{r, r+1} \neq 0, \quad r \in \mathbb{Z}_{+} .
$$

Thus, $\mathcal{M} \in \mathfrak{M}_{3}^{+}$and $A \in C_{+}(H)$. 
Remark 1. Condition (ii) of the last theorem may be replaced by the following condition which does not use a conjugation $J$ :

$(i i)^{*}$ there exists a cyclic vector $x_{0}$ of $A$ such that the following relations hold:

$$
\Gamma\left(x_{0}, x_{1}, \ldots, x_{n}, x_{n}^{*}\right)=0, \quad \forall n \in \mathbb{N},
$$

where

$$
x_{k}=A^{k} x_{0}, \quad x_{k}^{*}=\left(A^{*}\right)^{k} x_{0}, \quad k \in \mathbb{N},
$$

and the following operator:

$$
L \sum_{k=0}^{\infty} \alpha_{k} A^{k} x_{0}:=\sum_{k=0}^{\infty} \overline{\alpha_{k}}\left(A^{*}\right)^{k} x_{0}, \quad \alpha_{k} \in \mathbb{C},
$$

where all but finite number of coefficients $\alpha_{k}$ are zeros, is a bounded operator in $H$ which extends by continuity to a conjugation in $H$.

Let us show that conditions $(i),(i i) \Leftrightarrow$ conditions $(i),(i i)^{*}$.

The necessity is obvious since the conjugation $J$ satisfies relation (14) (with $J$ instead of $L$ ). Sufficiency. Let conditions $(i),(i i)^{*}$ be satisfied. Notice that

$$
\begin{aligned}
& L A A^{k} x_{0}=L A^{k+1} x_{0}=\left(A^{*}\right)^{k+1} x_{0}, \\
& A^{*} L A^{k} x_{0}=A^{*}\left(A^{*}\right)^{k} x_{0}=\left(A^{*}\right)^{k+1} x_{0}, \quad k \in \mathbb{Z}_{+} .
\end{aligned}
$$

By continuity we get $L A=A^{*} L$. Then condition (ii) holds with the conjugation $L$.

Remark 2. Notice that conditions (13) may be written in terms of the coordinates of $x_{0}$ in an arbitrary orthonormal basis $\left\{u_{n}\right\}_{n=0}^{\infty}$ in $H$ :

$$
x_{0}=\sum_{n=0}^{\infty} x_{0, n} u_{n}, \quad A^{k} x_{0}=\sum_{n=0}^{\infty} x_{0, n} A^{k} u_{n}, \quad\left(A^{*}\right)^{k} x_{0}=\sum_{n=0}^{\infty} x_{0, n}\left(A^{*}\right)^{k} u_{n} .
$$

By substitution these equalities in relation (8) we get some algebraic equations with respect to the coordinates $x_{0, n}$. If cyclic vectors of $A$ are unknown, one can use numerical methods to find approximate solutions of these equations. Then there should be cyclic vectors of $A$ among these solutions.

The following theorem gives an analogous description for the class $C_{-}(H)$.

Theorem 2. Let $A$ be a linear bounded operator in an infinite-dimensional Hilbert space $H$. The operator $A$ belongs to the class $C_{-}(H)$ if and only if the following conditions hold:

(i) A is a complex skew-symmetric operator with a simple spectrum;

(ii) there exists a cyclic vector $x_{0}$ of $A$ such that the following relations hold:

$$
\Gamma\left(x_{0}, x_{1}, \ldots, x_{n}, x_{n}^{*}\right)=0, \quad \forall n \in \mathbb{N}
$$

where

$$
x_{k}=A^{k} x_{0}, \quad x_{k}^{*}=\left(A^{*}\right)^{k} x_{0}, \quad k \in \mathbb{N} ;
$$

and $J x_{0}=x_{0}$, for a conjugation $J$ in $H$ such that $J A J=-A^{*}$. 
Condition (ii) of this theorem may be replaced by the following condition:

$(\text { ii })^{*}$ there exists a cyclic vector $x_{0}$ of $A$ such that relations (15), (16) hold and the following operator:

$$
L \sum_{k=0}^{\infty} \alpha_{k} A^{k} x_{0}:=\sum_{k=0}^{\infty}(-1)^{k} \overline{\alpha_{k}}\left(A^{*}\right)^{k} x_{0}, \quad \alpha_{k} \in \mathbb{C},
$$

where all but finite number of coefficients $\alpha_{k}$ are zeros, is a bounded operator in $H$ which extends by continuity to a conjugation in $H$.

The proof of the latter facts is similar and essentially the same as for the case of $C_{+}(H)$.

The following example shows that condition $(i i)$ (or $\left.(i i)^{*}\right)$ can not be removed from Theorem 1.

Example 1. Let $\sigma(\theta)$ be a non-decreasing left-continuous bounded function on $[0,2 \pi]$ with an infinite number of points of increase and such that

$$
\int_{0}^{2 \pi} \ln \sigma^{\prime}(\theta) d \theta=-\infty
$$

Consider the Hilbert space $L^{2}([0,2 \pi], d \sigma)$ of (classes of equivalence of) complex-valued functions $f(\theta)$ on $[0,2 \pi]$ such that

$$
\|f\|_{L^{2}([0,2 \pi], d \sigma)}^{2}:=\left(\int_{0}^{2 \pi}|f(\theta)|^{2} d \sigma(\theta)\right)^{\frac{1}{2}}<\infty .
$$

The condition (17) provides that algebraic polynomials of $e^{i \theta}$ are dense in $L^{2}([0,2 \pi], d \sigma)$ [5, p. 19]. Therefore the operator

$$
U f(\theta)=e^{i \theta} f(\theta), \quad f \in L^{2}([0,2 \pi], d \sigma),
$$

is a cyclic unitary operator in $H$, with a cyclic vector $f_{0}(\theta)=1$. Set

$$
J f(\theta)=\overline{f(\theta)}, \quad f \in L^{2}([0,2 \pi], d \sigma) .
$$

Then

$$
J U J f(\theta)=J e^{i \theta} \overline{f(\theta)}=e^{-i \theta} f(\theta)=U^{-1} f(\theta)=U^{*} f(\theta) .
$$

Thus, $U$ is a complex symmetric operator with a simple spectrum and condition $(i)$ of Theorem 1 is satisfied.

However, $U \notin C_{+}(H)$. In fact, suppose to the contrary that there exists an orthonormal basis $\left\{e_{j}\right\}_{j \in \mathbb{Z}_{+}}$such that the corresponding matrix $\mathcal{M}=\left(m_{k, l}\right)_{k, l=0}^{\infty}$ from (7) belongs to the class $\mathfrak{M}_{3}^{+}$. Since $U$ is unitary, we have

$$
\mathcal{E}=\mathcal{M M}^{*}
$$

with the usual rules of matrix operations, $\mathcal{E}=\left(\delta_{k, l}\right)_{k, l=0}^{\infty}$. However, the direct calculation shows that the element of the matrix $\mathcal{M M}^{*}$ in row 0 , column 2 is equal to $m_{0,1} \overline{m_{2,1}} \neq 0$. We obtained a contradiction. Thus, $U \notin C_{+}(H)$. Consequently, condition (ii) in Theorem 1 is essential and can not be removed.

Proposition 1. Let $H$ be an arbitrary infinite-dimensional Hilbert space. The class $C_{+}(H)$ is a proper subset of the set of all complex symmetric operators with a simple spectrum in $H$. 
Proof. Consider an arbitrary infinite-dimensional Hilbert space $H$. Let $V$ be an arbitrary unitary operator which maps $L^{2}([0,2 \pi], d \sigma)$ (see Example 1) onto $H$. Then $\widehat{U}:=V U V^{-1}$ is a unitary operator in $H$ with a simple spectrum and it has a cyclic vector $\widehat{x}_{0}:=V 1$. Since $J U J=U^{*}$, we get

$$
J V^{-1} \widehat{U} V J=V^{-1} \widehat{U}^{*} V, \quad V J V^{-1} \widehat{U} V J V^{-1}=\widehat{U}^{*} .
$$

Observe that $\widehat{J}:=V J V^{-1}$ is a conjugation in $H$. Therefore $\widehat{U}$ is a complex symmetric operator in $H$. Suppose that $\widehat{U} \in C_{+}(H)$. Let $\mathcal{F}=\left\{f_{k}\right\}_{k=0}^{\infty}$ be an orthonormal basis in $H$ such that the matrix $M=\left(m_{k, l}\right)_{k, l=0}^{\infty}, m_{k, l}=\left(\widehat{U} f_{l}, f_{k}\right)_{H}$, belongs to $\mathfrak{M}_{3}^{+}$. Observe that $\mathcal{G}=\left\{g_{k}\right\}_{k=0}^{\infty}$, $g_{k}:=V^{-1} f_{k}$, is an orthonormal basis in $L^{2}([0,2 \pi], d \sigma)$ and

$$
\left(U g_{l}, g_{k}\right)_{L^{2}([0,2 \pi], d \sigma)}=\left(V^{-1} \widehat{U} V g_{l}, g_{k}\right)_{L^{2}([0,2 \pi], d \sigma)}=\left(\widehat{U} f_{l}, f_{k}\right)_{H}=m_{k, l}, \quad k, l \in \mathbb{Z}_{+} .
$$

Therefore $U \in C_{+}\left(L^{2}([0,2 \pi], d \sigma)\right)$. This is a contradiction with Example 1. Consequently, we have $\widehat{U} \notin C_{+}(H)$.

On the other hand, the class $C_{+}(H)$ is non-empty, since an arbitrary matrix from $\mathcal{M}_{3}^{+}$with bounded elements define an operator $B$ in $H$ which have this matrix in an arbitrary fixed orthonormal basis in $H$.

Remark 3. The classical Jacobi matrices are closely related to orthogonal polynomials [4]. Let us indicate some similar relations for the class $\mathfrak{M}_{3}^{+}$. Choose an arbitrary $\mathcal{M}=\left(m_{k, l}\right)_{k, l=0}^{\infty} \in \mathfrak{M}_{3}^{+}$, where $m_{k, l} \in \mathbb{C}$. Let $\left\{p_{n}(\lambda)\right\}_{n=0}^{\infty}, \operatorname{deg} p_{n}=n, p_{0}(\lambda)=1$, be a sequence of polynomials defined recursively by the following relation:

$$
m_{n, n-1} p_{n-1}(\lambda)+m_{n, n} p_{n}(\lambda)+m_{n, n+1} p_{n+1}(\lambda)=\lambda p_{n}(\lambda), \quad n=0,1,2, \ldots,
$$

where $m_{0,-1}:=1, p_{-1}:=0$. Set $c_{n}=m_{n, n+1}, b_{n}=m_{n, n}, n \in \mathbb{Z}_{+} ;$and $c_{-1}:=1$. By (5), (18) we get

$$
c_{n-1} p_{n-1}(\lambda)+b_{n} p_{n}(\lambda)+c_{n} p_{n+1}(\lambda)=\lambda p_{n}(\lambda), \quad n=0,1,2, \ldots
$$

Let $p_{n}(\lambda)=\mu_{n} \lambda^{n}+\cdots, \mu_{n} \in \mathbb{C}, n \in \mathbb{Z}_{+}$. Comparing coefficients by $\lambda^{n+1}$ in (19) we get

$$
\mu_{n+1}=\frac{1}{c_{n}} \mu_{n}, \quad n \in \mathbb{Z}_{+} .
$$

By induction we see that

$$
\mu_{n}=\left(\prod_{j=0}^{n-1} c_{j}\right)^{-1}, \quad n \in \mathbb{N}, \quad \mu_{0}=1
$$

Set

$$
P_{n}(\lambda)=\prod_{j=0}^{n-1} c_{j} p_{n}(\lambda), \quad n \in \mathbb{N}, \quad P_{0}(\lambda)=1, \quad P_{-1}(\lambda)=0 .
$$

Multiplying the both sides of (19) by $\prod_{j=0}^{n-1} c_{j}, n \geq 1$, we obtain:

$$
c_{n-1}^{2} P_{n-1}(\lambda)+b_{n} P_{n}(\lambda)+P_{n+1}(\lambda)=\lambda P_{n}(\lambda), \quad n=0,1,2, \ldots
$$


By Theorem 6.4 in [6] there exists a complex-valued function $\phi$ of bounded variation on $\mathbb{R}$ such that

$$
\int_{\mathbb{R}} P_{m}(\lambda) P_{n}(\lambda) d \phi(\lambda)=\left(\prod_{j=0}^{n-1} c_{j}\right)^{2} \delta_{m, n}, \quad m, n \in \mathbb{Z}_{+} .
$$

Therefore we get

$$
\int_{\mathbb{R}} p_{m}(\lambda) p_{n}(\lambda) d \phi(\lambda)=\delta_{m, n}, \quad m, n \in \mathbb{Z}_{+}
$$

Polynomials $\left\{p_{n}(\lambda)\right\}_{n=0}^{\infty}$ were used in [7,8] to state and solve the direct and inverse spectral problems for matrices from $\mathfrak{M}_{3}^{+}$. Analogs of some facts of the Weyl discs theory were obtained for the case of matrices from $\mathfrak{M}_{3}^{+}$with additional assumptions [9]: $m_{n, n+1}>0, n \in \mathbb{Z}_{+}$, and

$$
m_{n, n} \in \mathbb{C}: \quad r_{0} \leq \operatorname{Im} m_{n, n} \leq r_{1},
$$

for some $r_{0}, r_{1} \in \mathbb{R}, n \in \mathbb{Z}_{+}$.

On the other hand, the direct and inverse spectral problems for matrices from $\mathfrak{M}_{3}^{-}$were investigated in [10].

Probably, some progress in the spectral theory of complex symmetric and skew-symmetric operators would provide some additional information about corresponding polynomials and vice versa.

\section{Acknowledgements}

The author is grateful to referees for their comments and suggestions.

\section{References}

[1] Garcia S.R., Putinar M., Complex symmetric operators and applications, Trans. Amer. Math. Soc. 358 (2006), 1285-1315.

[2] Garcia S.R., Putinar M., Complex symmetric operators and applications. II, Trans. Amer. Math. Soc. 359 (2007), 3913-3931.

[3] Zagorodnyuk S.M., On a $J$-polar decomposition of a bounded operator and matrices of $J$-symmetric and $J$-skew-symmetric operators, Banach J. Math. Anal. 4 (2010), no. 2, 11-36.

[4] Akhiezer N.I., Classical moment problem, Fizmatlit, Moscow, 1961 (in Russian).

[5] Geronimus Ya.L., Orthogonal polynomials on the circle and on an interval. Estimates, asymptotic formulas, orthogonal series, Fizmatlit, Moscow, 1958 (in Russian).

[6] Chihara T.S., An introduction to orthogonal polynomials, Mathematics and its Applications, Vol. 13, Gordon and Breach Science Publishers, New York - London - Paris, 1978.

[7] Guseinov G.Sh., Determination of an infinite non-self-adjoint Jacobi matrix from its generalized spectral function, Mat. Zametki 23 (1978), 237-248 (English transl.: Math. Notes 23 (1978), 130-136).

[8] Zagorodnyuk S.M., Direct and inverse spectral problems for $(2 N+1)$-diagonal, complex, symmetric, nonHermitian matrices, Serdica Math. J. 30 (2004), 471-482.

[9] Zagorodnyuk S.M., Integral representations for spectral functions of some nonself-adjoint Jacobi matrices, Methods Funct. Anal. Topology 15 (2009), 91-100.

[10] Zagorodnyuk S.M., The direct and inverse spectral problems for $(2 N+1)$-diagonal complex transpositionantisymmetric matrices, Methods Funct. Anal. Topology 14 (2008), 124-131. 